\title{
Construction of the simulation model "car- railway track" for modeling the dynamic loading process of the boot node
}

\author{
Igor Martinov", Alyona Trufanova, and Vadim Shovkun \\ Ukrainian State University of Railway Transport, Car Department, 61050, Kharkiv, Ukraine
}

\begin{abstract}
Using of software systems in the design of new samples is increasingly important, but there is a problem in adapting computer models to obtain adequate results of calculations. The modern development of the IT systems gives the opportunity to significantly reduce the timing of the introduction of new equipment into production by reducing the number of field experimental test samples of the design technology. Every year using of complexes software in designing new samples is becoming increasingly important, but there is a problem in adapting computer models to obtain adequate results of calculations. UM Universal Mechanism complex was used to simulate the dynamic loading process of the boot node. Modelling of dynamic loadings on the running gears of freight cars showed a sufficient convergence with the results of running tests, about $87 \%$. Therefore, the proposed model can be used for assessing of disturbing loads when the calculating of the reliability of the bucket units of freight cars was done.
\end{abstract}

The safety of the movement of rolling stock depends on the reliability of aggregates, assemblies and parts of cars [1].

The reliability of the car is conditioned by its reliability, durability and the longevity of its parts. Therefore there is a need to increase the reliability of the rolling stock by allocating of sufficient resources for the current content and implementation of new principles of repair and elimination of the consequences in emergency situations, additional investment in new technologies, equipment modernization and development of methods aimed at improving operational the activity of cars.

The modern development of the IT systems gives the opportunity to significantly reduce the timing of the introduction of new equipment into production by reducing the amount of field experimental research samples of the design technology.

Every year, using of complex software in designing new samples is becoming increasingly important, but there is a problem in adapting computer models to obtain adequate results of calculations. UM Universal Mechanism complex was used to simulate the dynamic loading process of the boot node. The simulation model "railroad car" was developed by UM. The mathematical model includes geometric, inertial, stiffness and friction characteristics.

* Corresponding author: vadimshovkun62@gmail.com 
The features of the model allow:

- to study indicators of dynamic characteristics (coefficients of dynamics of spring sets, vertical and transverse accelerations of separate points of a body, etc.);

- to investigate the safety indicators (coefficients of stability of wheels against the return of rails, frame and lateral forces, axial load, etc.);

- vary by main geometric, rigid, frictional and inertial parameters of the car and spring suspension;

- to investigate the movement of a car at different speeds with various wear of running parts, wheel profiles and rails, rigidity and inertia characteristics of the track with irregularities;

- to model the movement of cars with various structural modifications of trolleys.

A freight car consists of 19 solid bodies ( 4 wheel pairs with booms, 4 side frames, 8 friction wedges, 2 spindle beams, and 1 body) and their corresponding 114 degrees of freedom. Fig. 1 shows the design scheme of such a freight car.

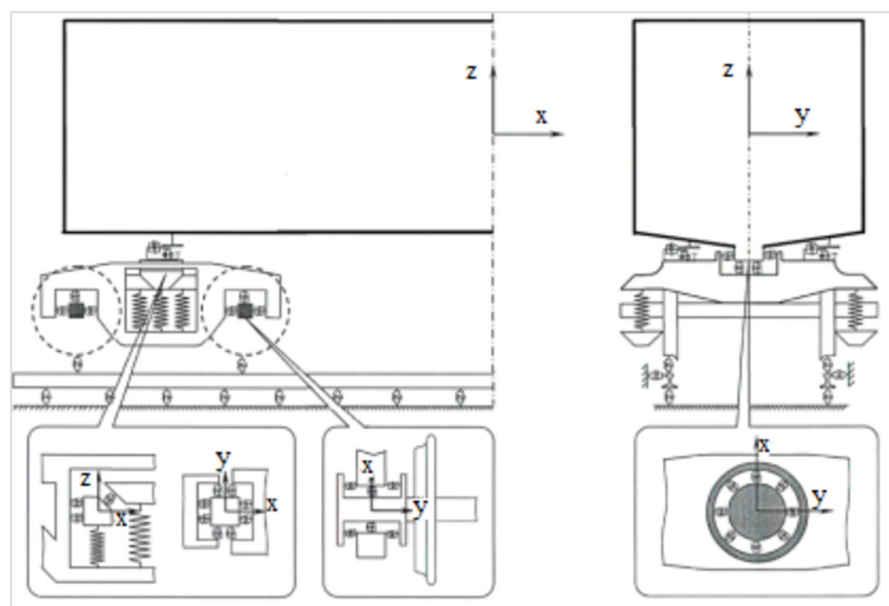

Fig. 1. Estimated power scheme of the car and railway track

In the mathematical model of UM software complex for each solid is given the mass $m i$ and moments of inertia $\mathrm{Ji}$ to its own body coordinate system (CKi) \{Ci,xi,yi,zi\} Its beginning coincides with the centre of mass Fig. 2. Two general systems of coordinates are introduced to the scheme: the fixed base one (CKOO) $\{C O, X 0, Y O, Z O\}$ and the base flexible one (track) (CKO) $\{0, \xi, \eta, \zeta\}$.

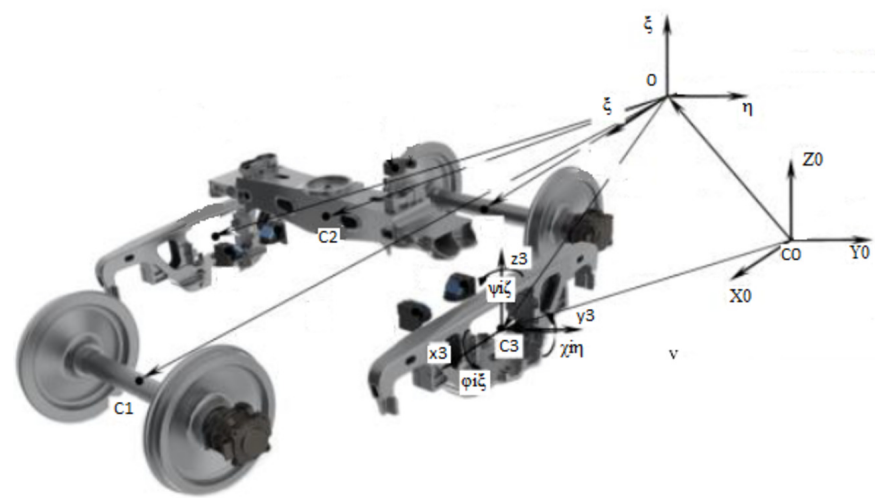

Fig. 2. Body coordinate system. 
The movement of the car is presented as a complex movement. The moving base (track) coordinate system moves progressively along the axis of the railroad with the speed of the crew. The start of the JMA is associated with the centre of gravity of the bodies. The fixed system $C K O$ determines the position of the rail path in space. The movement of the car is presented as a complex movement.

The moving base (track) coordinate system moves progressively along the axis of the railroad with the speed of the crew. The start of the JMA is associated with the centre of gravity of the bodies. The fixed system $C K O O$ determines the position of the rail path in space.

The position of the $i$-th body in the $C K O$ is determined by the position of the vector ri (0) (the lower index marks the body number for which the value is calculated, and the upper index shows in which coordinate system the calculation is made) connects the start of the $C K O$ and $C K i$, and the angles between axes of $C K O$ and $S K I$. These values can be represented as a vector tensor of the vector on the $C K O(\mathbf{R i}(0)$ axis) and the rotation matrix (cosine paths) $(A i(0))$.

In the UM complex, the kinematic characterization of the body system is described by a programmatic mathematical procedure and is implemented as a hinge linking this body with the base coordinate system $[2,3]$.

The spatial orientation of each body included in the model of the freight car is given by its own system of coordinates parallel to the axes $C K O$. In the process of hinge description a vector ri ( 0$)$ for each body in the initial position (for $t=0$ ) is given. Thus, the position of the car bodies is set in the system of the railroad crew. If necessary, for any body, the number of degrees of freedom may be reduced by prohibiting the change of translational or rotational coordinates. At this stage, with the help of the problem of inertial and geometric quantities, parametrization of the mathematical model through various identifiers was carried out.

Based on the theorem about the motion of the centre of masses and the dynamic Euler equation, when the $C K s$ coincide with the principal axes of inertia of the bodies the differential equations are obtained by UM software complex which describe the motion of the i-th body of the mathematical model, which have the form:

$$
\left\{\begin{array}{l}
\operatorname{mi} \cdot \operatorname{aix}(0)=\sum \operatorname{Fix}(0)+\sum \operatorname{Rix}(0) ; \\
\operatorname{mi} \cdot \operatorname{aiy}(0)=\sum \operatorname{Fiy}(0)+\sum \operatorname{Riy}(0) ; \\
\operatorname{mi} \cdot \operatorname{aiz}(0)=\sum \operatorname{Fiz}(0)+\sum \operatorname{Riz}(0) ; \\
\operatorname{Jix}(i) \cdot d \omega i x(i) d t+\omega i y(i) \omega i z(i) \cdot(\operatorname{Jiz}(i)-\operatorname{Jiy}(i))=\sum \operatorname{Mix}(i)+\sum \operatorname{Lix}(i) ; \\
\operatorname{Jiy}(i) \cdot d \omega i y(i) d t+\omega i z(i) \omega i x(i) \cdot(\operatorname{Jix}(i)-\operatorname{Jiz}(i))=\sum \operatorname{Miy}(i)+\sum \operatorname{Liy}(i) ; \\
\operatorname{Jiz}(i) \cdot d \omega i z(i) d t+\omega i x(i) \omega i y(i) \cdot(\operatorname{Jiy}(i)-\operatorname{Jix}(i))=\sum \operatorname{Miz}(i)+\sum \operatorname{Liz}(i)
\end{array}\right.
$$

$m i$ - mass of the $i$-th body, $H$;

aix (0), aiy (0), aiz(0) of the acceleration vector of the $i$-th body on the coordinate axis CKO, $\mathrm{m} / \mathrm{s}^{2}$;

$\Sigma F i x(0), \Sigma F i y(0), \Sigma F i z(0)$ is the sum of the projections of all active forces applied to the $i$-th body, on the coordinate axes $C K O, H$;

$\Sigma \operatorname{Rix}(0), \Sigma R i y(0), \Sigma R i z(0)$ the sum of the projections of all reactive forces applied to the ith body, on the coordinate axes $C K O, H$;

$\operatorname{Jix}(i), J i y(i), J i z(i)$ moments of inertia of the $i$-th body relative to the axes $C K i, H / m 2$;

$\omega i x(i), \omega i y(i), \omega i z(i)$ projections of the angular velocity vector of the $i$-th body on the coordinate axes $S K i, \mathrm{rad} / \mathrm{s}$; 
$\Sigma M i x(i), \Sigma M i y(i), \Sigma M i z(i)$ is the sum of the moments of all active forces applied to the $i$ th body, relative to the axes $C K i, H / m$;

$\Sigma \operatorname{Lix}(i), \Sigma \operatorname{Liy}(i), \Sigma \operatorname{Liz}(i)$ is the sum of moments of all reactive forces applied to the $i$-th body, relative to the axes $C K i, H / M$.

The initial results of the simulation of dynamic modelling of loadings became the implementations which characterize the change in the coefficient of vertical and horizontal dynamics for a running wheel in a loaded and empty mode at a speed of $40-120 \mathrm{~km} / \mathrm{h}$, some implementations are shown in Fig. 3.

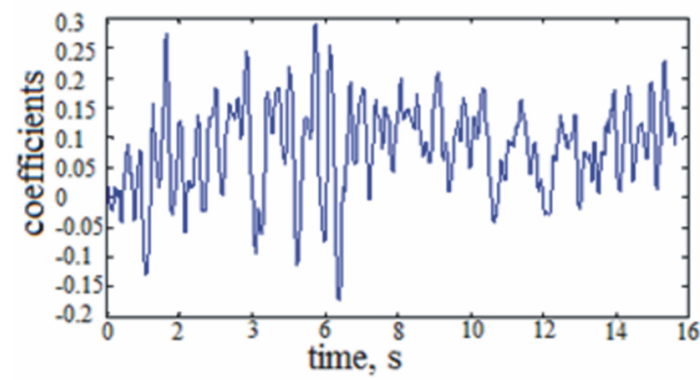

Fig. 3. Oscillogram of changes in the coefficient of vertical dynamics at a speed of $100 \mathrm{~km} / \mathrm{h}$.

Obviously, the process of time-varying coefficient of vertical dynamics is a random process with a pronounced sinusoidal component [4-10].

Similar conclusions can be drawn about the coefficients of horizontal dynamics: the simulation showed that there is a growth in accordance with the increase in speed. In load mode, these ratios did not exceed the normative values. In empty mode there was a slight excess of dependence on the velocity of the coefficients of vertical dynamics shown in Fig. 4.

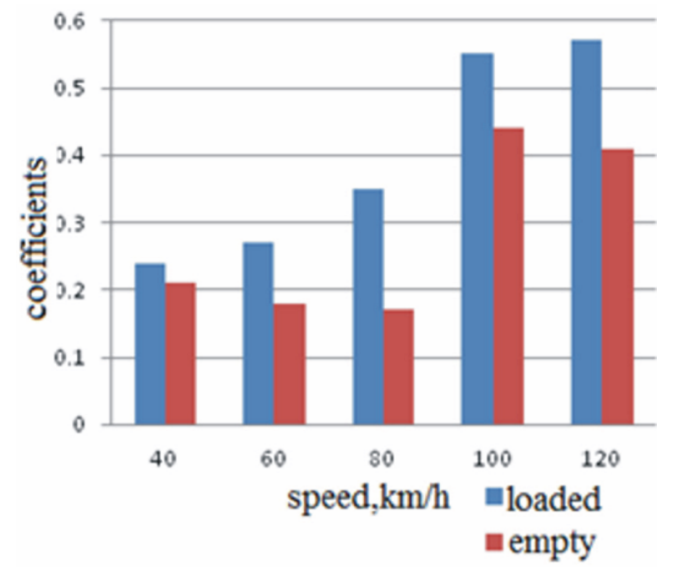

Fig.4. Dependence of the coefficient of vertical dynamics.

In the speed range from 11.1 to $33.3 \mathrm{~m} / \mathrm{s}(40 \div 120 \mathrm{~km} / \mathrm{h})$, the ranges of the change in the trolley were from 0.24 to 0.54 for the empty mode, which is below the permissible value of 0.9 .

\section{Conclusions}

1. Modelling of dynamic loadings on the running gears of freight cars showed a sufficient convergence with the results of running tests, about $87 \%$. 
2. Therefore, the proposed model can be used to estimate disturbing loads when calculating the reliability of the bucket units of freight cars.

3. The obtained data from the simulation can be used to calculate the reliability of the boot bearing nodes.

\section{References:}

1. I. Martynov, V. Ilchishin, East-European Journal of Advanced Technologies, 73, (2015)

2. E. Bratchev, V. Kamayev, Abstracts of the report of the All-Union Conference. Dnipropetrovsk From 97, (1984)

3. A. Savoskin, A. Akishin, Computer modeling in railway transport: dynamics, durability, wear. A collection of abstracts, Bryansk 69, (2016)

4. I. Martynov, A. Trufanova, V. Shovkun, Computer modeling in railway transport: dynamics, durability, wear. A collection of abstracts, Bryansk, 44, (2016)

5. G. Mikhalchenko, D. Pogorelov, V. Simonov, Dnipropetrovsk National University of Railway Transport, 107, (1996)

6. Simson, E. Bulletin of the NTU "KhPI", 37, 142, (2010)

7. Averin, N. Vestnik VNIIZhT, 3, 24, (2007)

8. Sanchugov V. Scientific journal "Izvestiya Samara Scientific Center of the Russian Academy of Sciences", 831, (2007)

9. Gishvarov, A. Vestnik of the Ufa State aviation technical university, 1, 43, (2007)

10. Haydamaka, A. Visnyk of Dnipropetrovsk National University of Railway Transport, 3, 51, (2014) 